MATHEMATICS OF COMPUTATION

Volume 74, Number 251, Pages 1217-1230

S 0025-5718(04)01688-6

Article electronically published on September 10, 2004

\title{
A CONVERGENT DIFFERENCE SCHEME FOR THE INFINITY LAPLACIAN: CONSTRUCTION OF ABSOLUTELY MINIMIZING LIPSCHITZ EXTENSIONS
}

\author{
ADAM M. OBERMAN
}

\begin{abstract}
This article considers the problem of building absolutely minimizing Lipschitz extensions to a given function. These extensions can be characterized as being the solution of a degenerate elliptic partial differential equation, the "infinity Laplacian", for which there exist unique viscosity solutions.

A convergent difference scheme for the infinity Laplacian equation is introduced, which arises by minimizing the discrete Lipschitz constant of the solution at every grid point. Existence and uniqueness of solutions to the scheme is shown directly. Solutions are also shown to satisfy a discrete comparison principle.

Solutions are computed using an explicit iterative scheme which is equivalent to solving the parabolic version of the equation.
\end{abstract}

\section{INTRODUCTION}

In this article we introduce and implement a convergent finite difference scheme for the "infinity Laplacian" partial differential equation (PDE). The infinity Laplacian was studied by Aronsson [3] in the late 1960s and has been the subject of renewed interest in recent work by Barles and Busca, Crandall, Evans, Gariepy, Jensen, and others. There has also been a recent comprehensive study by Aronsson, Crandall, and Juutinen [5. Solutions of the infinity Laplacian are in a certain sense the best solutions of the classical analysis problem of extending a Lipschitz function. The infinity Laplacian is also the Euler-Lagrange equation for the prototypical problem in the calculus of variations in $L^{\infty}$. The problem has applications both to other areas of mathematics, for example in the classical transportation problem of Monge [14, and outside of mathematics, for example in image processing and engineering mechanics.

The problem requires good numerical analysis, since naive solution methods may be numerically stable, but nevertheless fail to converge. The scheme we present respects at the discrete level two essential features: the comparison principle and the absolutely minimizing Lipschitz extension property.

Received by the editor September 30, 2003 and, in revised form, December 29, 2003.

2000 Mathematics Subject Classification. Primary 35B50, 35J60, 35J70, 65N06, 65N12.

Key words and phrases. Finite difference, infinity Laplacian, viscosity solution.

The author would like to thank P. E. Souganidis and A. Petrosyan for valuable discussions and L. C. Evans for his encouragement and enthusiasm. 
Consider then

$$
\Delta_{\infty} u=\frac{1}{|D u|^{2}} \sum_{i, j=1}^{m} u_{x_{i} x_{j}} u_{x_{i}} u_{x_{j}}=0
$$

for $x$ in a bounded, open set $U$ in $\mathbb{R}^{m}$, along with Dirichlet boundary conditions $u=g$ on the boundary of $U$. Here $|D u|=\left(u_{x_{1}}^{2} \ldots u_{x_{m}}^{2}\right)^{1 / 2}$ is the length of the gradient of the function $u$. (The definition above is the one which arises naturally in our discretization schemes, but it is different from the one used by some authors, who may omit the factor of $1 /|D u|^{2}$.) A more suggestive form is to rewrite the equation as the second derivative in the direction of the gradient,

$$
\Delta_{\infty} u=\frac{d^{2} u}{d v^{2}}, \quad \text { where } v=\frac{D u}{|D u|} .
$$

The infinity Laplacian operator appears in the equation for the motion of level sets by mean curvature, $\Delta_{1} u=\Delta u-\Delta_{\infty} u$, where $\Delta_{1}$ is the level set mean curvature operator, and $\Delta$ is the usual Laplacian.

The scheme is defined by locally minimizing the discrete Lipschitz constant of the solution, which leads to a one-dimensional, nonsmooth convex optimization problem, to be solved at each grid point. This optimization problem may be interpreted as the minimization of the relaxed gradient. An explicit solution of the optimization problem is found, which is then used to produce a consistent, monotone scheme. Convergence (as the grid parameters go to zero) to the viscosity solution of (III) follows from Barles-Souganidis [7]. The discretized problem is solved iteratively, using an explicit scheme that is equivalent to the explicit Euler discretization of $u_{t}=\Delta_{\infty} u$. This scheme is a contraction in $L^{\infty}$; consequently, the iterations converge exponentially to the solution.

In the next few paragraphs we discuss the infinity Laplacian PDE in more detail, connections with other areas of mathematics, and some applications. The contents of the remainder of article follow at the end of the section.

The classical problem of Lipschitz extensions. A classical problem in real analysis is to extend a given function to a larger domain without increasing its Lipschitz constant. Given the function $g$ defined on a closed set $C \subset \mathbb{R}^{m}$, with $K$ the least constant for which

$$
f(x)-f(y) \leq K|x-y| \text { for all } x, y \in C
$$

holds, the problem is to build a Lipschitz continuous extension of $g$ with the smallest possible Lipschitz constant. There are multiple solutions to the Lipschitz extension problem. The Whitney [20] and McShane [17] extensions,

$$
\Phi(x)=\inf _{y \in C}(f(y)+K|x-y|), \quad \Lambda(x)=\sup _{y \in C}(f(y)-K|x-y|),
$$

are both solutions. In fact they are the maximal and minimal extensions, respectively. Unfortunately, the McShane and Whitney extensions do not have certain desirable properties. For example, repeated applications of the operators may locally improve the Lipschitz constant [5]. 
Absolute minimizers and calculus of variations in $L^{\infty}$. Aronsson [2, 3, 4] studied the Lipschitz extension problem under the stronger requirement that the extensions be "absolutely minimizing". Given boundary data $g$, on $\partial U$, the extension $u$ is minimizing if $|D u|_{L^{\infty}(U)} \leq|D v|_{L^{\infty}(U)}$ for all other extensions $v$. A more stringent requirement is that a function be absolutely minimizing, which means that it is minimizing on every open, bounded subset of $U$.

Aronsson interpreted (IL) as the formal limit, as $p \rightarrow \infty$, of the problem of minimizing the functional $I_{p}(u)=\int_{U}|D u|^{p} d x$ under given boundary conditions. The Euler-Lagrange equation for $I_{p}$ is

$$
\operatorname{div}\left(|D u|^{p-2} D u\right)=|D u|^{p-2}\left(\Delta u+(p-2) \Delta_{\infty} u\right),
$$

which results in (IL), when $p \rightarrow \infty$.

The problem of finding absolute minimizers of $|D u|_{\infty}$ is the prototypical problem in the calculus of variations in $L^{\infty}$. Barron, Jensen and Wang [8, 9] prove the existence of absolute minimizers and derive the corresponding Aronsson-Euler equations for more general problems in the calculus of variations in $L^{\infty}$.

Existence and uniqueness of viscosity solutions. The function $f(x, y)=|x|^{4 / 3}-$ $|y|^{4 / 3}$ is an example, due to Aronsson [4], of a function which is absolutely minimizing, but not twice differentiable. As of the date of this article, the differentiability of solutions of (IL) remains an open question.

Since there exist nonsmooth solutions to (IL), solutions cannot be interpreted in the classical sense. Viscosity solutions 13 provide a suitable notion of weak solution. Uniqueness of viscosity solutions to (IL) was first proved by Jensen [16. Later, using different techniques, uniqueness results were established in [6] and [5]. A geometric definition of solutions is provided by the notion of comparison with cones. Equivalence of the viscosity solution definition, the comparison with cones definition, and the absolutely minimizing Lipschitz property are established in 12 .

Applications to engineering mechanics. The minimization of $|D u|$ in the $L^{1}$ and $L^{\infty}$ norms under given boundary data is studied by Strang in [19]. According to Strang, the $L^{\infty}$ and $L^{1}$ norms appear naturally in many engineering problems. Applications include the minimization of the maximum stress and the minimization of the maximum deflection in an elastic system. Duality theory in convex analysis is used to give equivalent formulations of the problems.

Applications to image processing. Degenerate elliptic PDE have appeared in image processing, and more recently in image interpolation (inpainting). Axioms for image processing were introduced and analyzed in 1]. Any image transform which satisfies certain reasonable axioms can be represented as the solution operator for a degenerate parabolic PDE. The infinity Laplacian operator (understood as the second derivative in the direction of the gradient) has also been used for edge detection in the image processing community since as far back as 1984; see [11] for references.

Applications of (IL) and other PDEs to image interpolation (rather than image processing) are discussed in [11. In a parallel fashion to image processing, a class of degenerate elliptic PDEs arise. The class includes the mean curvature operator (1Laplacian), the usual Laplacian, and the infinity Laplacian. Computational results are presented for the image interpolation problem, using an ad hoc algorithm to solve (IL). 
Interpolation operators which do not satisfy the axioms may be flawed. A minimal Lipschitz interpolation algorithm is presented in [10, which fails to be absolutely minimizing. Consequently, as observed in Caselles et al., the interpolant may be discontinuous.

Summary of main results and overview of contents. An explicit, convergent finite difference scheme is presented for the infinity Laplace equation. The scheme arises at the solution of the discrete absolutely minimizing Lipschitz extension problem. The scheme is defined on a finite difference grid with two parameters, the spatial resolution $d x$ and the directional resolution $d \theta$. As $d x$ and $d \theta$ go to 0 independently, the solution of the scheme converges to the viscosity solution of (IL), with formal accuracy $O(d x+d \theta)$.

In 1.1 we present an approximation scheme for (IL) in a simple case. In 2 we recall the notion of discrete ellipticity for finite difference schemes. In $\$ 3$ we recall the definitions of viscosity solutions for (IL), consistency for approximation schemes and the convergence result. In $\$ 4$ the scheme is defined and shown to be consistent and convergent. In $\$ 5$ numerical validation and examples are presented.

1.1. The scheme in a special case. If the neighboring grid points are equidistant, for example on a uniform hexagonal grid, the resulting approximation scheme is simpler. We present this special case here. In order to define a scheme on a rectilinear grid, we need the general case, which will be presented in $\$ 4$

Let $B_{r}(y)$ denote the ball of radius $r$ centered at $y$ and define $\widehat{v}=v /|v|$. For a smooth function $u$,

$$
\begin{aligned}
& u(x+r \widehat{D u})=\max _{x \in \partial B_{r}(y)} u(x)+O\left(r^{2}\right), \\
& u(x-r \widehat{D u})=\min _{x \in \partial B_{r}(y)} u(x)+O\left(r^{2}\right) .
\end{aligned}
$$

Taking into account (1.1) gives the discretization

$$
-\Delta_{\infty} u(y)=\frac{1}{r^{2}}\left(2 u(x)-\min _{x \in \partial B_{r}(y)} u(x)-\max _{x \in \partial B_{r}(y)} u(x)\right)+O\left(r^{2}\right) .
$$

Given points on the sphere $x_{1}, \ldots, x_{n} \in \partial B_{r}(y)$, we may discretize further to obtain

$$
-\Delta_{\infty} u(y)=\frac{1}{r^{2}}\left(2 u(y)-\min _{i=1}^{n} u\left(x_{i}\right)-\max _{i=1}^{n} u\left(x_{i}\right)\right)+O\left(r^{2}+d \theta\right),
$$

where $d \theta$ is a measure of the error in going from (1.2) to (1.3); see $\$ 4$

The connection between absolutely minimizing Lipschitz extensions and the infinity Laplace equation in a discrete setting is given by the following observations.

A convex optimization problem. To build a scheme on a cartesian grid, (1.3) must be generalized to allow for nonequidistant neighbors. This is accomplished by solving the discrete optimal Lipschitz extension problem. Minimize the (discrete) Lipschitz constant of $u$ at $x_{0}$ computed with respect to the points $x_{1}, \ldots, x_{n}$ :

$$
\min _{u_{0}} L\left(u_{0}\right), \quad \text { where } L\left(u_{0}\right)=\max _{i=1}^{n} \frac{\left|u\left(x_{0}\right)-u\left(x_{i}\right)\right|}{\left|x_{0}-x_{i}\right|},
$$


which is a convex, nonsmooth one-dimensional optimization problem. The solution in the equidistant case is simply the average of the max and the min of the values

$$
u_{*}=\frac{1}{2}\left(\min _{i=1}^{n} u\left(x_{i}\right)+\max _{i=1}^{n} u\left(x_{i}\right)\right) .
$$

This solution appears in (1.3): $-\Delta_{\infty} u=2 r^{-2}\left(u-u_{*}\right)+O\left(r^{2}+d \theta\right)$. In the general case, the minimizer is found by maximizing the relaxed (discrete) gradient

$$
\max _{i, j=1}^{n} \frac{\left|u\left(x_{i}\right)-u\left(x_{j}\right)\right|}{\left|x_{0}-x_{i}\right|+\left|x_{0}-x_{j}\right|}
$$

over the neighboring nodes and linearly interpolating the values at the maximal nodes; see Theorem 5

\section{DisCRETELY ELLiPtiC FINITE DIFFERENCE SCHEMES}

In this section we review some background material on monotone finite difference schemes. The discretization of (IL) gives a system of coupled, nonlinear (nondifferentiable) equations. In general, these types of systems may be quite challenging to solve, but fortunately, discretely elliptic discretizations yield systems which may be solved by a simple explicit iteration scheme.

We begin with an informal description of the comparison principle and the degenerate ellipticity property for PDEs to motivate the parallel definitions of monotonicity and the discrete ellipticity property for numerical schemes. This is followed by formal definitions and a statement of the main theorems, the full details of which may be found in 18 .

It is convenient for heuristic purposes to regard the solution operator of a PDE as a map from "data" to "solution" (where "data" may represent suitable boundary conditions and "solution" may represent a function on the domain). In this context, the comparison principle is a global property of the solution operator which says: if "data $1 \leq$ data2", then "solution $1 \leq$ solution2." Here the operator, $\leq$, is the natural partial ordering on functions. Degenerate ellipticity is a local structure condition on the PDE which is used to prove the comparison principle.

In a parallel fashion, it is convenient to regard the solution operator for an approximation scheme as a map taking "data" to "solution" (where now the data and the solution may be a finite number of function values). In this context, monotonicity is a global property which says: if "data1 $\leq$ data2", then "solution1 $\leq$ solution2." Discrete ellipticity, introduced in [18, is a local structure condition on the approximation scheme which is used to prove monotonicity.

2.1. Definitions and theorems. A finite difference grid is a graph with $N$ vertices embedded in $\mathbb{R}^{m}$. Given a node $i$, write $e(i)=\left\{i_{1}, \ldots i_{n_{i}}\right\}$ for the list of its neighbors. We identify vectors $\mathbf{u}=\left(u_{1}, \ldots, u_{N}\right) \in \mathbb{R}^{N}$ with functions on the grid and call them grid functions.

Definition (Discretely elliptic finite difference scheme). A function $\mathcal{F}: \mathbb{R}^{N} \rightarrow \mathbb{R}^{N}$, which is regarded as a map from grid functions to grid functions, is a finite difference scheme if

$$
\mathcal{F}(\mathbf{u})^{i}=\mathcal{F}^{i}\left(u_{i}, u_{i}-u_{i_{1}}, \ldots, u_{i}-u_{i_{n_{i}}}\right) \quad(i=1, \ldots, N)
$$


for some functions $\mathcal{F}^{i}\left(x_{0}, x_{1}, \ldots, x_{n_{i}}\right)$. A grid function $\mathbf{u}$ is a solution of the scheme, if $\mathcal{F}(\mathbf{u})=\mathbf{0}$. The scheme $\mathcal{F}$ is discretely elliptic if

$$
x \leq y \text { implies that } \mathcal{F}^{i}(x) \leq \mathcal{F}^{i}(y), \quad \text { for } i=1, \ldots, N \text {. }
$$

Note that when applied to a grid vector, discrete ellipticity means that $\mathcal{F}^{i}$ is nondecreasing in $u_{i}$ and in the differences $u_{i}-u^{\prime}$. (To emphasize the parallel nature of the definitions, recall that the nonlinear PDE $F\left(D^{2} u, D u, u, x\right)$ is degenerate elliptic if it is nondecreasing in the $u$ argument and nonincreasing in the $D^{2} u$ argument.)

Definition (Explicit Euler map and nonlinear CFL condition). Let $\mathcal{F}$ be a Lipschitz continuous, discretely elliptic scheme, with Lipschitz constant $K$. For $\rho>0$, define the explicit Euler map $\mathcal{S}_{\rho}: \mathbb{R}^{N} \rightarrow \mathbb{R}^{N}$ by

$$
\mathcal{S}_{\rho}(\mathbf{u})=\mathbf{u}-\rho \mathcal{F}(\mathbf{u}) .
$$

The nonlinear Courant-Freidrichs-Lewy condition for $\mathcal{S}_{\rho}$ is

$$
\rho \leq \frac{1}{K} .
$$

Uniformly/strictly proper are mild technical conditions that may be satisfied without loss of generality by adding a small multiple (say $d x$ ) of $u_{i}$ to each component $\mathcal{F}^{i}$ of the scheme.

Theorem 1 (Discretely elliptic schemes are monotone). Let $\mathcal{F}$ be a strictly proper, discretely elliptic finite difference scheme. Then $\mathcal{F}$ is monotone.

Theorem 2 (Monotonicity of the Euler map). Let $\mathcal{F}$ be a Lipschitz continuous, discretely elliptic scheme. The Euler map (2.3) is monotone provided (CFL) holds.

Thus given a discretely elliptic scheme for an elliptic PDE, the explicit Euler discretization in time gives a monotone scheme for the corresponding parabolic PDE. (It is also true that the implicit Euler discretization is discretely elliptic.)

Theorem 3 (Contractivity of the Euler map). Let $\mathcal{F}$ be a Lipschitz continuous, discretely elliptic scheme. The Euler map (2.3) is a contraction in $\mathbb{R}^{N}$ equipped with the maximum norm, provided (CFL) holds. If, in addition, $\mathcal{F}$ is uniformly proper and strict inequality holds in (CFD), then the Euler map is a strict contraction.

Theorem 4 (Construction of unique solutions). Every uniformly proper, Lipschitz continuous discretely elliptic scheme has a unique solution. The iterates of the explicit Euler map converge exponentially to this solution for arbitrary initial data, as long as strict inequality holds in (CFL).

\section{VisCOSITY SOLUTIONS}

In this section we recall the definition of viscosity solutions for the infinity Laplacian and the definition of consistency used in the convergence theory.

Definition (Viscosity solutions). (1) A continuous function $u$ defined on the set $U$ is a viscosity subsolution of $-\Delta_{\infty} u=0$ in $U$, if for every local minimum point $x \in U$ of $u-\phi$, where $\phi$ is $C^{2}$ in some neighborhood of $x$, we have

$$
\begin{cases}-\Delta_{\infty} \phi(x) \leq 0, & \text { if } D \phi(x) \neq 0, \\ -\eta^{T} D^{2} \phi(x) \eta \leq 0, & \text { for some }|\eta| \leq 1, \text { if } D \phi(x)=0 .\end{cases}
$$


(2) A continuous function $u$ defined on the set $U$ is a viscosity supersolution of $-\Delta_{\infty} u=0$ in $U$, if for every local maximum point $x \in U$ of $u-\phi$, where $\phi$ is $C^{2}$ in some neighborhood of $x$, we have

$$
\begin{cases}-\Delta_{\infty} \phi(x) \geq 0, & \text { if } D \phi(x) \neq 0, \\ -\eta^{T} D^{2} \phi(x) \eta \geq 0, & \text { for some }|\eta| \leq 1, \text { if } D \phi(x)=0 .\end{cases}
$$

(3) Moreover, a continuous function defined on the set $U$ is a viscosity solution of $-\Delta_{\infty} u=0$ in $U$, if it is both a viscosity subsolution and a viscosity supersolution in $U$.

Definition (Consistency). The numerical scheme $\mathcal{F}^{d x, d \theta}$ is consistent if for every $\phi \in C^{2}(U)$ and for every $x \in U$,

$$
\lim _{d x, d \theta \rightarrow 0} \mathcal{F}^{d x, d \theta}(\phi)(x)=-\Delta_{\infty} \phi(x)
$$

if $D \phi(x) \neq 0$, and

$$
\lambda \leq \liminf _{d x, d \theta \rightarrow 0} \mathcal{F}^{d x, d \theta}(\phi)(x) \leq \limsup _{d x, d \theta \rightarrow 0} \mathcal{F}^{d x, d \theta}(\phi)(x) \leq \Lambda,
$$

where $\lambda, \Lambda$ are the least and greatest eigenvalues of $D^{2} \phi(x)$, otherwise.

By a theorem of Barles-Souganidis [7], consistent, monotone schemes converge to the unique viscosity solution of the PDE.

\section{Discrete minimal Lipschitz eXtensions}

In this section we define and solve the discrete minimal Lipschitz extension problem, which will then be used to define the approximation scheme.

We mention that the discrete minimal Lipschitz extension problem is formulated for points in Euclidean space, but the arguments apply equally to points in a metric space. This approach may be useful for more general problems, for example inpainting on a surface or extending a function in a metric space.

Definition (Discrete Lipschitz constant). Given distinct points $x_{0}, \ldots, x_{n}$ in $\mathbb{R}^{m}$, let $d_{i}=\left|x_{0}-x_{i}\right|>0, i=1, \ldots, n$, be the distances from the base point $x_{0}$ to the neighbors. Given a function $u$ defined in a neighborhood containing the points $x_{i}, i=0, \ldots, n$, let $u_{i}=u\left(x_{i}\right)$, for $i=1, \ldots n$. The discrete Lipschitz constant of the function $u$, at the point $x_{0}$, computed with respect to the points $x_{1}, \ldots, x_{n}$, is

$$
L\left(u_{0}\right)=\max _{i=1}^{n} L^{i}\left(u_{0}\right)=\max _{i=1}^{n} \frac{\left|u_{0}-u_{i}\right|}{d_{i}} .
$$

Problem 1 (Discrete minimal Lipschitz extensions). Minimize the discrete Lipschitz constant of $u$ at $x_{0}$, computed with respect to the points $x_{1}, \ldots, x_{n}$ over the value $u_{0}=u\left(x_{0}\right)$ :

$$
\min _{u_{0}} L\left(u_{0}\right)
$$

Remark (Geometrical interpretation of Problem 1). Consider $\mathbb{R}^{n}$ imbued with the metric $d(x, y)=\max _{i=1}^{n}\left|x_{i}-y_{i}\right| / d_{i}$, where $d_{i}>0, i=1, \ldots, n$. Then Problem 1 consists of finding the minimum distance from the point $\left(u\left(x_{1}\right), \ldots, u\left(x_{n}\right)\right)$ to the diagonal line $(t, \ldots, t), t \in \mathbb{R}$. 
Theorem 5 (Solution of the discrete minimal Lipschitz extension problem). The unique solution of the discrete minimal Lipschitz extension problem is

$$
u_{*}=\frac{d_{i} u_{j}+d_{j} u_{i}}{d_{i}+d_{j}},
$$

where $i, j$ are the indices which maximize the relaxed discrete gradient

$$
\frac{\left|u_{i}-u_{j}\right|}{d_{i}+d_{j}}=\max _{k, l=1}^{n}\left\{\frac{\left|u_{k}-u_{l}\right|}{d_{k}+d_{l}}\right\} .
$$

Furthermore,

$$
u_{*} \text { is nondecreasing as a function of }\left(u_{1}, \ldots, u_{n}\right) \text {. }
$$

Proof. The function $L(u): \mathbb{R} \rightarrow \mathbb{R}$ is convex and piecewise linear, with $L(u)^{\prime} \neq 0$ on each piece, so $L(u)$ has a unique minimum, $u_{*}$. The minimum occurs at the unique point where $\lim _{u \uparrow u_{*}} L^{\prime}(u)<0$ and $\lim _{u \downarrow u_{*}} L^{\prime}(u)>0$. Rewrite

$$
L(u)=\max _{i, j=1}^{n} L^{i, j}(u)
$$

where

$$
L^{i, j}(u)=\max \left\{\frac{\left|u-u_{i}\right|}{d_{i}}, \frac{\left|u-u_{j}\right|}{d_{j}}\right\} .
$$

By the description (4.5), all possible vertices of $L$ are vertices of $L^{i, j}$, for some indices $i, j$. Thus the minimum of $L$ occurs at a minimum of $L^{i, j}$ for some $i, j$. Therefore

$$
\min _{u} \max _{i, j=1}^{n} L^{i, j}(u)=\max _{i, j=1}^{n} \min _{u} L^{i, j}(u) .
$$

The minimum of $L^{i, j}(u)$ occurs when $\left(u-u_{i}\right) / d_{i}=\left(u-u_{j}\right) / d_{j}$. This gives a minimum at the linear interpolant

$$
u_{i j}=\frac{d_{i} u_{j}+d_{j} u_{i}}{d_{i}+d_{j}}
$$

with value

$$
L(u)=L^{i j}\left(u_{i j}\right)=\frac{\left|u_{i}-u_{j}\right|}{d_{i}+d_{j}} .
$$

Taking into account the minimax property (4.6) gives (4.2), (4.3).

Next, we check the assertion (4.4). Write $u^{\prime}$ for $u_{1}, \ldots, u_{n}$. Now $L(u)=L\left(u, u^{\prime}\right)$ : $\mathbb{R}^{n+1} \rightarrow \mathbb{R}$ is a continuous function, convex in $u$ for each fixed $u^{\prime}$. So the minimum $u_{*}=u_{*}\left(u^{\prime}\right)$ is a continuous function of $u^{\prime}$. In addition, $u_{*}$ is piecewise linear by (4.7). Since on each piece, $u_{*}$ is nondecreasing in $u^{\prime}$, it follows from continuity that $u_{*}$ is globally nondecreasing in $u^{\prime}$, which establishes (4.4).

The scheme can now be defined using the solution of the discrete minimal Lipschitz extension problem at each point.

Definition (Scheme definition). Define the scheme at each grid point to be $u_{0}-u_{*}$ where $u_{*}=u_{*}\left(u_{1}, \ldots, u_{n}\right)$ is the solution of the discrete minimal Lipschitz extension problem, computed with respect to neighbors. 
The scheme is in the form (2.1), since it can be written

$$
u_{0}-u_{*}=\frac{d_{i} d_{j}}{d_{i}+d_{j}}\left(\frac{u_{0}-u_{j}}{d_{j}}+\frac{u_{0}-u_{i}}{d_{i}}\right),
$$

where $i, j$ are the indices defined by (4.3).

We remark that it is the expression $u-u_{*}$, not $\left(u-u_{*}\right) /\left(d_{i} d_{j}\right)$ which is discretely elliptic, so in fact the scheme computes $d_{i} d_{j} \Delta_{\infty} u$ at each grid point, where $i, j$ may vary. This is acceptable, since the scheme nevertheless finds solutions of $\Delta_{\infty}=0$. In addition, the Lipschitz constant of the scheme is unity, which is also convenient.

Next we prove consistency.

Definition (Spatial and directional resolution). Given distinct points, $x_{0}, x_{1}, \ldots$, $x_{n}$ in $\mathbb{R}^{m}$, define the direction vectors $v_{i}=x_{0}-x_{i}$, the distances $d_{i}=\left|v_{i}\right|$, and the unit direction vectors $\hat{v}_{i}=v_{i} / d_{i}$. Define the local spatial resolution

$$
d x=\max _{i=1}^{n}\left|v_{i}\right|
$$

and the local directional resolution

$$
d \theta=\max _{v \in S^{n}} \min _{i=1}^{n}\left|v-\hat{v}_{i}\right|,
$$

which is a bound on the distance from an arbitrary direction vector (unit vector) to the set of grid direction vectors. Finally, define the variation in the distances,

$$
d x^{\prime}=\max _{i=1}^{n} d_{i}-\min _{i=1}^{n} d_{i} .
$$

In the theorem below we will assume

$$
\begin{aligned}
& \text { if } \hat{v}_{i} \text { is a direction vector, then so is }-\hat{v}_{i}, \\
& \qquad d x^{\prime}=O(1),
\end{aligned}
$$

simply to ensure that the $d_{i}$ are interchangeable with $d x$ in the computations below.

Theorem 6 (Consistency of discrete minimal Lipschitz extensions). Let $u$ be $a$ $C^{2}$ function in a neighborhood of $x_{0}$. Suppose we are given neighbors $x_{1}, \ldots, x_{n}$, arranged symmetrically so that (4.11) holds and so that the mild technical condition (4.12) holds. Let $u_{*}$ be the solution of the discrete minimal Lipschitz extension problem computed with respect to the points $x_{1}, \ldots, x_{n}$, and let $i, j$ be the indices which maximize the relaxed discrete gradient (4.3). Then

$$
-\Delta_{\infty} u\left(x_{0}\right)=\frac{1}{d_{i} d_{j}}\left(u\left(x_{0}\right)-u_{*}\right)+O(d \theta+d x) .
$$

Proof. 1. First assume $D u \neq 0$. The Taylor expansion gives

$$
u_{i}=u\left(x_{0}\right)+d_{i} \hat{v}_{i} D u\left(x_{0}\right)+\frac{d_{i}^{2}}{2} \hat{v}_{i}^{T} D^{2} u\left(x_{O}\right) \hat{v}_{i}+O\left(d x^{3}\right), \quad i=1, \ldots, n .
$$

Then (4.3), which is to be maximized, gives

$$
\frac{u_{k}-u_{l}}{d_{k}+d_{l}}=\left(\hat{v}_{k}-\hat{v}_{l}\right) D u+O(d x) \text {. }
$$

Up to order $d x$, the last expression is maximized by choosing $\hat{v}_{k}$ as close as possible to $\widehat{D u}$ and $\hat{v}_{l}$ close to $-\widehat{D u}$. Furthermore, taking $d x, d \theta$ small enough, by (4.11) we may assume that

$$
\hat{v}_{i}=-\hat{v}_{j}
$$


By assumption, there are indices $i, j$ so that

$$
\left|\hat{v}_{i}-\widehat{D u}\right|,\left|\hat{v}_{j}+\widehat{D u}\right| \leq d \theta
$$

Then using the Taylor expansion (4.14) and the solution formula (4.2) gives

$$
u_{*}=u_{0}+\frac{d_{i} d_{j}}{d_{i}+d_{j}}\left(\hat{v}_{i}+\hat{v}_{j}\right) D u+\frac{1}{2} d_{i} d_{j}\left(\hat{v}_{i} D^{2} u \hat{v}_{i}+\hat{v}_{j} D^{2} u \hat{v}_{j}\right)+O\left(d x^{3}\right)
$$

and using (4.15) and applying (4.16), we get (4.13), as desired.

2. Next, assume $D u=0$. Then for any indices $i, j$, we have from (4.14)

$$
\frac{1}{d_{i} d_{j}}\left(u_{0}-\frac{d_{j} u_{i}+d_{i} u_{j}}{d_{i}+d_{j}}\right)=\frac{1}{2}\left(\hat{v}_{i} D^{2} u \hat{v}_{i}+\hat{v}_{j} D^{2} u \hat{v}_{j}\right)+O(d x)
$$

which is consistent up to $O(d x)$ with $-\Delta_{\infty} u$ in the viscosity sense.

Theorem 7 (Convergence). The solution of the difference scheme defined above converges (uniformly on compact sets) as $d x, d \theta$ goes to 0 to the solution of (III).

Proof. Convergence to the solution of (IL) follows from consistency (Theorem 6) and monotonicity (Theorem [5) and Theorem 1 1 of the scheme by [7].

\section{IMPLEMENTATION}

In this section, the numerical implementation of the scheme is discussed, and numerical solutions are presented. Solutions are displayed in Figure 2

The scheme was implemented on a uniform rectilinear grid, in two dimensions. The directional resolution $d \theta$ was reduced by increasing the number of neighbors associated with each node. This was accomplished as follows. Taking as neighbors four nodes on the boundary of the square of radius $d x$ gave the five point scheme. Taking as neighbors all nine nodes on the boundary of the square of radius $d x$ gave the nine point scheme. Finally, taking as neighbors all sixteen nodes on the boundary of the square of radius $2 d x$ gave the seventeen point scheme. See Figure 1
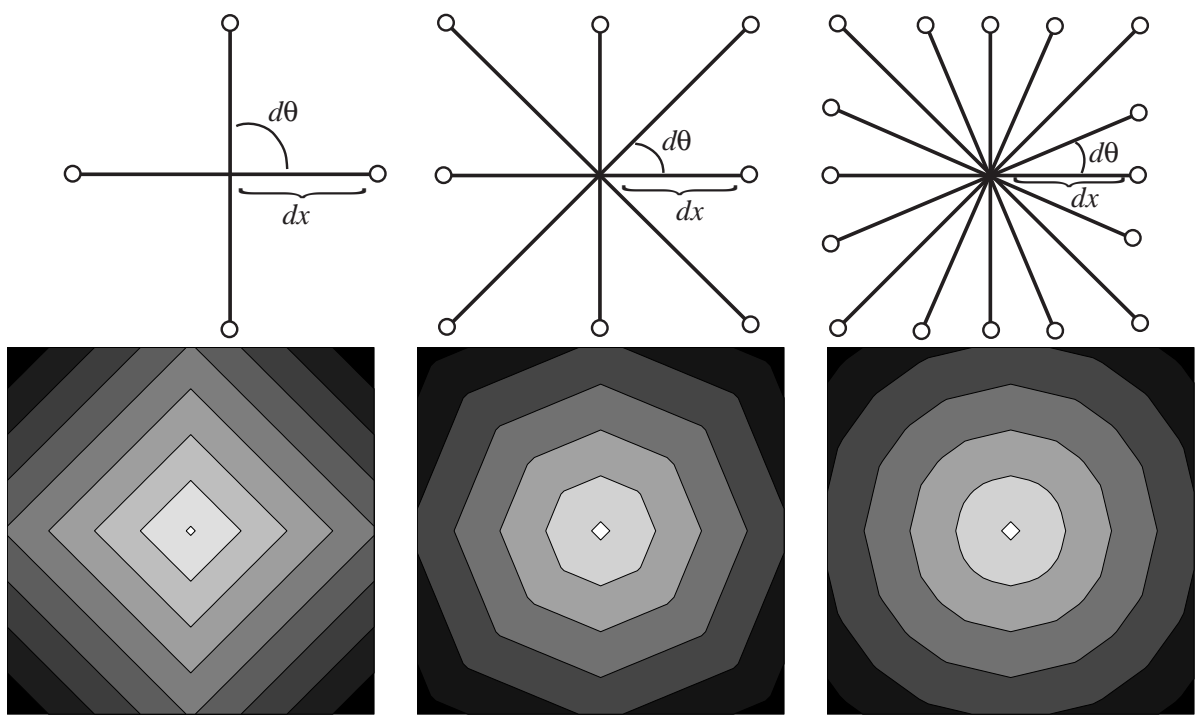

Figure 1. Grids for the 5, 9, and 17 point schemes and level sets of the cones for the corresponding schemes. 
TABLE 1. Discretization error as a function of $d \theta$, computed using neighbors on the boundary of a circle, and on a square, for different choices of quadratic functions.

\begin{tabular}{|c|c|c|c|c|c|c|c|c|}
\hline Circle: \# points & 16 & \multicolumn{2}{|r|}{32} & 64 & 200 & \multicolumn{2}{|c|}{1000} & $10^{5}$ \\
\hline error & .1553 & & 0123 & .0 .123 & -0.016 & & 024 & -.00008 \\
\hline Square: \# levels & 2 & 4 & 8 & 16 & 32 & 64 & 128 & 256 \\
\hline error q1 & .11 & .11 & .11 & -.11 & .05 & .027 & -.013 & .0068 \\
\hline error q2 & -.2 & -.2 & -.2 & .05 & -.004 & -.004 & -.004 & .0015 \\
\hline
\end{tabular}

Near the boundary, the seventeen point scheme was modified to also include points on the boundary of the square of radius $d x$, which gave a twenty-five point scheme; this was needed for continuity at the boundary.

Each choice of neighbors induces a natural metric on the grid. The cones in the induced metric were computed by solving $|D u|_{\mathcal{G}}=1$, using the discretely elliptic discretization, $|D u|_{\mathcal{G}}=\max _{i=1}^{n_{i}}\left\{\left(u_{0}-u_{i}\right) / d_{i}\right\}$. The level sets of the cones are shown in Figure 1.

Nonconvergent methods. In preliminary investigations, several other methods were attempted, all of which failed to converge. A naive difference method for (ID) is to first compute the gradient and then compute the second derivative of the function in the direction determined by the gradient. This method violates the comparison principle: increasing the value of the function at one of the grid points used to compute the gradient will change the resulting direction, and in general the resulting value of the second derivative is not greater. Implementing this method led to spurious finite amplitude oscillations in the solutions. Other methods were either insensitive to sharp corners (failing to see that a cone with interior vertex is not a solution) or produced spurious sharp saddles of the approximate form $|x|-|y|$.

Discretization error. An independent check of the discretization error was performed for data on the unit circle and also for data on the nodes of a uniform box. Quadratic data was assigned to the grid points, and the Lipschitz extension problem was solved to compute $u-u_{*}$. Data was deliberately chosen so that the gradients did not line up with the grid. The observed accuracy, presented in Table 1 was linear in $d \theta$, confirming the accuracy analysis.

For the calculations on the square, lattice points on the boundary of the square of radius $d x$ times the number of levels were used. For the calculations on the circle, equally spaced points on the boundary of the circle of radius $d x$ were used.

TABLE 2. Numerical error computed in the maximum norm for the 5 point, 9 point, and 25 point stencils, on a grid with $n^{2}$ points. Calculated using the exact Aronsson solution $x^{4 / 3}-y^{4 / 3}$.

\begin{tabular}{|c|l|l|l|l|l|}
\hline Stencil & $n=41$ & $n=81$ & $n=161$ & $n=241$ & $n=401$ \\
\hline 5 & .09 & .09 & .067 & .015 & .0057 \\
\hline 9 & .023 & .018 & .0058 & .0028 & .00079 \\
\hline 25 & .0052 & .0057 & .0033 & .0035 & .00072 \\
\hline
\end{tabular}


Numerical convergence. The observed convergence was much better than predicted by the analysis: the observed error was linear in $d x$, even for fixed $d \theta$, as evidenced by Table 2 where the error was computed using the exact solution $x^{4 / 3}-y^{4 / 3}$.

Implementation. The scheme was implemented on a uniform grid. The number of grid points used varied from $41^{2}$ to $401^{2}$. The implementation was performed in MATLAB, on a laptop computer. The entire code was less than two hundred lines. Convergence was defined to be when $u-u_{*}<d x / 200$ at each node. Convergence required on the order of 1000 iterations. Convergence time was less than a minute of computer time for $n=100$ and several minutes for $n=401$. Solutions are displayed in Figure 2. Solutions appear differentiable, but not twice differentiable.
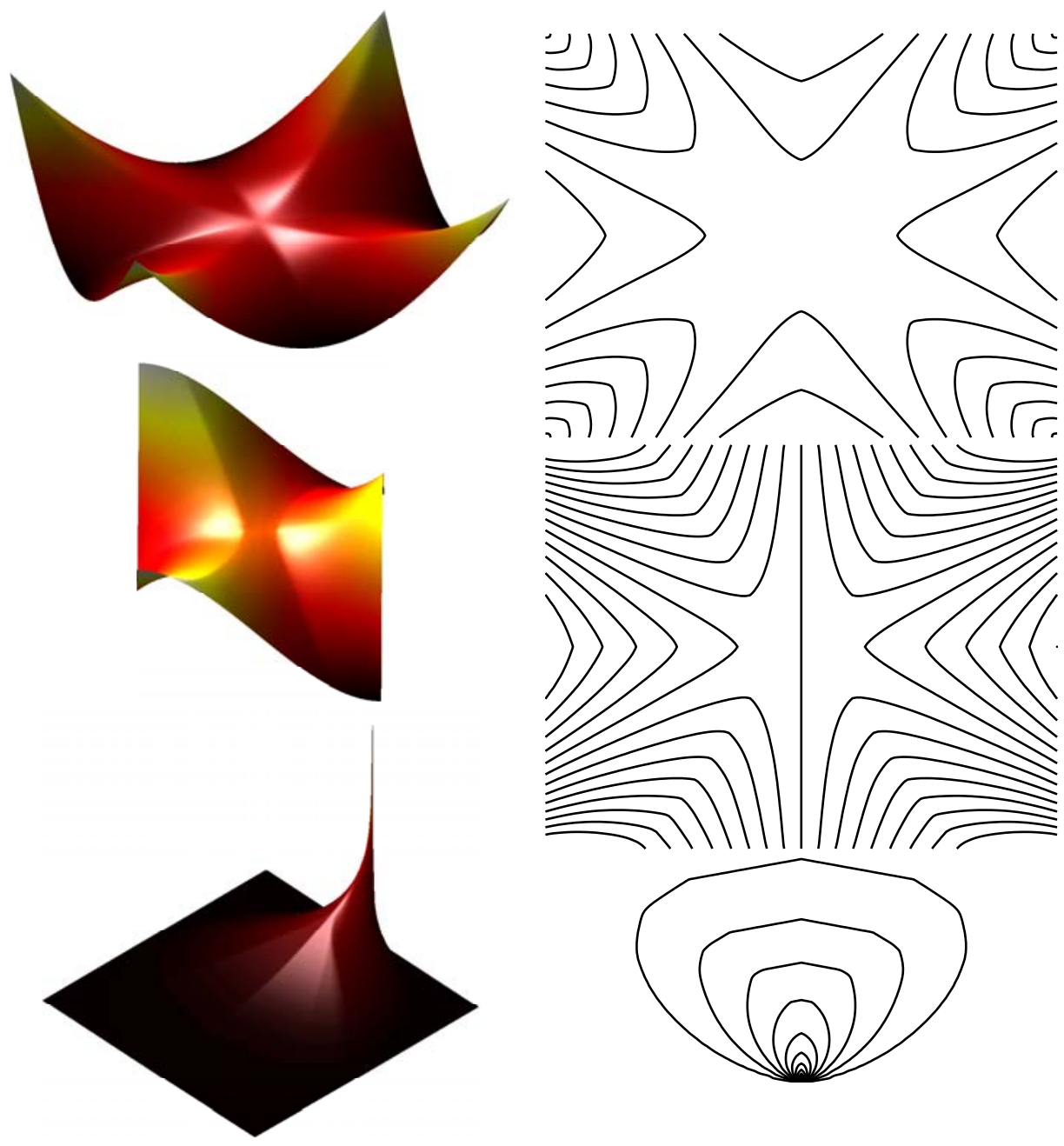

Figure 2. Surface and contour plots using the 25 neighbor method, for boundary data $|x|$, and $x^{3}-3 x y^{2}$, on a square, and boundary data the characteristic function of a point, on a circle. 
The singularities are visualized using the lighting effects. Singularities appeared along lines which meet at saddle points. The kinks in the solution are not numerical artifacts. The third solution shown is for boundary data the characteristic function of a point, on a circle. The problem has been studied by Evans [15. The contours have corners which reflect the metric on the grid. As $d \theta \rightarrow 0$, we expect the corners to vanish.

The logic of the scheme is such that the number of neighbors determined how the local Lipschitz constant was minimized. Thus boundary nodes (nodes with zero neighbors and Dirichlet data assigned) could be placed anywhere. Likewise, nodes on the boundary of the computational domain were simply nodes with fewer neighbors and need not have Dirichlet data assigned.

As observed in 1.1 the scheme simplifies when the neighboring grid points are equidistant. An implementation using a hexagonal grid would be simpler: the resulting iteration requires simply replacing the value at each point by the average of the min and max of its neighbors.

\section{REFERENCES}

1. Luis Alvarez, Frédéric Guichard, Pierre-Louis Lions, and Jean-Michel Morel. Axioms and fundamental equations of image processing. Arch. Rational Mech. Anal., 123(3):199-257, 1993. MR 94j:68306

2. Gunnar Aronsson. Extension of functions satisfying Lipschitz conditions. Ark. Mat., 6:551-561 (1967), 1967. MR 36:754

3. Gunnar Aronsson. On the partial differential equation $u_{x}^{2} u_{x x}+2 u_{x} u_{y} u_{x y}+u_{y}{ }^{2} u_{y y}=0$. Ark. Mat., 7:395-425 (1968), 1968. MR.38:6239

4. Gunnar Aronsson. On certain singular solutions of the partial differential equation $u_{x}^{2} u_{x x}+$ $2 u_{x} u_{y} u_{x y}+u_{y}^{2} u_{y y}=0$. Manuscripta Math., 47(1-3):133-151, 1984. MR85m:35011

5. Gunnar Aronsson, Michael G. Crandall, and Petri Juutinen. A tour of the theory of absolutely minimizing functions. 65 pages, http:www.math.ucsb.edu/ crandall/paperdir/index.html, July 2003.

6. Guy Barles and Jérôme Busca. Existence and comparison results for fully nonlinear degenerate elliptic equations without zeroth-order term. Comm. Partial Differential Equations, 26(1112):2323-2337, 2001. MR 2002k:35078

7. Guy Barles and Panagiotis E. Souganidis. Convergence of approximation schemes for fully nonlinear second order equations. Asymptotic Anal., 4(3):271-283, 1991. MR.92d:35137

8. Emmanuel N. Barron, Robert R. Jensen, and Changyou Wang. The Euler equation and absolute minimizers of $L^{\infty}$ functionals. Arch. Ration. Mech. Anal., 157(4):255-283, 2001. MR2002m:49006

9. Emmanuel N. Barron, Robert R. Jensen, and Changyou Wang. Lower semicontinuity of $L^{\infty}$ functionals. Ann. Inst. H. Poincaré Anal. Non Linéaire, 18(4):495-517, 2001. MR:2002c:49020

10. Josep R. Casas and Luis Torres. Strong edge features for image coding, pages 443450. Kluwer, Boston, MA, May 1996. R.W. Schafer, P. Maragos, and M.A. Butt, Eds. http:citeseer.nj.nec.com/113454.html.

11. Vicent Caselles, Jean-Michel Morel, and Catalina Sbert. An axiomatic approach to image interpolation. IEEE Trans. Image Process., 7(3):376-386, 1998. MR.2000d:94001

12. M. G. Crandall, Lawrence C. Evans, and R. F. Gariepy. Optimal Lipschitz extensions and the infinity Laplacian. Calc. Var. Partial Differential Equations, 13(2):123-139, 2001. MR2002h:49048

13. Michael G. Crandall, Hitoshi Ishii, and Pierre-Louis Lions. User's guide to viscosity solutions of second order partial differential equations. Bull. Amer. Math. Soc. (N.S.), 27(1):1-67, 1992. MR92j:35050

14. Lawrence C. Evans and Wilfrid Gangbo. Differential equations methods for the MongeKantorovich mass transfer problem. Mem. Amer. Math. Soc., 137(653):viii+66, 1999. MR99g:35132

15. Lawrence C. Evans. Personal communication. 
16. Robert Jensen. Uniqueness of Lipschitz extensions: minimizing the sup norm of the gradient. Arch. Rational Mech. Anal., 123(1):51-74, 1993. MR.94g:35063

17. Edward James McShane. Extension of range of functions. Bull. Amer. Math. Soc., 40:837-842, 1934.

18. Adam M. Oberman. Convergent difference schemes for degenerate elliptic and parabolic equations: Hamilton-Jacobi equations and free boundary problems. http:www.math. utexas.edu/ oberman, 2003.

19. Gilbert Strang. $L^{1}$ and $L^{\infty}$ approximation of vector fields in the plane. In Nonlinear partial differential equations in applied science (Tokyo, 1982), volume 81 of North-Holland Math. Stud., pages 273-288. North-Holland, Amsterdam, 1983. MR85c:49010

20. Hassler Whitney. Analytic extensions of differentiable functions defined in closed sets. Trans. Amer. Math. Soc., 36(1):63-89, 1934.

Department of Mathematics, Simon Fraser University, 8888 University Dr., Burnaby, British Columbia Canada V5A 1 S6

E-mail address: aoberman@sfu.ca 